\title{
Hallazgo de Aedes aegypti (Linnaeus 1762), en el casco urbano del corregimiento de La Pedrera, Amazonas, Colombia
}

\author{
Yesika Rojas-Gil', Helena Brochero ${ }^{2,3}$ \\ ${ }_{1}^{1}$ Programa de Enfermedades Transmitidas por Vectores, Secretaría de Salud de Amazonas, Leticia, \\ Amazonas. \\ ${ }^{2}$ Laboratorio de Entomología, Instituto Nacional de Salud, Bogotá, D.C., Colombia. \\ ${ }^{3}$ Facultad de Agronomía, Universidad Nacional de Colombia, Bogotá, D.C., Colombia.
}

Introducción. Hasta el año 2005 no se había registrado la especie Aedes aegypti en el departamento de Amazonas en Colombia. En el marco de la vigilancia entomológica para enfermedades transmitidas por vectores realizada por la Secretaría de Salud Departamental, se registra en abril de 2006 la presencia de este mosquito en el casco urbano del corregimiento de La Pedrera, Amazonas.

Objetivo. Registrar el hallazgo de Ae. aegypti en el departamento de Amazonas.

Materiales y métodos. Se realizaron levantamientos de índices de infestación larvaria para Ae. aegypti y se calcularon los valores clásicos de índice de vivienda (IV) (porcentaje de casas que presentaron criaderos con larvas), índice de depósito (ID) (porcentaje de depósitos con larvas con respecto al total de depósitos inspeccionados) y el índice de Breteau (IB) (número de depósitos con larvas de la especie en 100 viviendas inspeccionadas). Se indagó a la comunidad sobre la percepción de la presencia del insecto en sus viviendas. Se realizaron actividades de control tendientes a reducir la infestación del mosquito.

Resultados. Se registró Ae. aegypti en el casco urbano del corregimiento de La Pedrera. Durante la primera inspección los valores de los índices correspondieron a: IV=29,6\%; ID=9,0\%; $\mathrm{IB}=40,8 \%$. Se observó disminución de estos valores luego de las actividades de control de vectores realizadas. Sin embargo, no se eliminó la infestación de la especie en esta área geográfica. La comunidad reconoce las larvas del mosquito en los recipientes de su domicilio $\mathrm{y}$, en general, lo asocia como transmisor de diversas enfermedades.

Conclusión. Este estudio evidenció el hallazgo de Ae. aegypti en el departamento de Amazonas. En el casco urbano de La Pedrera no se encontró Ae. albopictus.

Palabras clave: Aedes aegypti, vigilancia, dengue, fiebre amarilla, Colombia.

New record of Aedes aegypti(Linnaeus, 1762), in the urban area of La Pedrera, Amazonas, Colombia

Introduction. Before 2005, Aedes aegypti had not been recorded in the Colombian province of Amazonas. Because this species has been increasing its range throughout Colombia, an entomological surveillance program has been routinely directed toward detecting it presence in Amazonas by the Amazonas State Health Authority.

Objective. Surveillance data were used as the basis for recording Aedes aegypti in Amazonas Province.

Materials and methods. Household surveys of Ae. aegypti larvae were conducted by trained personnel belonging to the Amazonas State Health Authority. The following standard larval indices were estimated: house index $(\mathrm{HI})$ - the percentage of premises with positive containers, the container index $(\mathrm{Cl})$-the percentage of positive containers among the water-holding containers, and the Breteau index (BI) - the number of positive containers per 100 premises. Residents were questioned concerning their perception of mosquito presence in their houses. Subsequently, control activities were undertaken to reduce the mosquito's infestation.

Results. Aedes aegypti was found in the urban area of the village of La Pedrera. During the first household survey, the indices were $\mathrm{HI}=29.6 \%, \mathrm{Cl}=9.0 \%$ and, $\mathrm{BI}=40.8 \%$. After control activities, 
these values decreased; however, elimination of the infestation was not possible in this geographical area. The community recognizes the mosquito immature forms in water containers in houses and associated them as vectors of several diseases.

Conclusion. The presence of Ae. aegypti mosquitoes in the village La Pedrera was verified. Aedes albopictus was not found in this area.

Key words: Aedes aegypti, surveillance, dengue, yellow fever, Colombia.

El departamento del Amazonas en Colombia hace parte de la cuenca amazónica y limita con Perú y Brasil. Cuenta con dos municipios, Leticia, capital departamental y Puerto Nariño. Además, se compone de nueve corregimientos: Tarapacá, La Chorrera, Puerto Santander, Mirití-Paraná, El Encanto, Puerto Arica, Puerto Alegría, La Victoria y La Pedrera (1).

El corregimiento de La Pedrera se ubica en el norte del departamento del Amazonas y limita con el departamento del Vaupés (figura 1). Según los registros del Grupo de Control de Enfermedades Transmitidas por Vectores de la Secretaría de Salud Departamental del Amazonas, 2007, cuenta con 3.138 habitantes, quienes se encuentran en 18 asentamientos sobre la margen del río Caquetá (incluyendo el casco urbano) y 21 comunidades en la ribera del río Apaporis. Según el boletín del censo general nacional $2005,99,5 \%$ de las viviendas de La Pedrera son casas, de las cuales, $19,4 \%$ tiene servicio de energía eléctrica, 2,4\% tiene acueducto y alcantarillado y $81,2 \%$ de la población residente se autorreconoce como indígena (2).

El departamento del Amazonas constituye un área endémica para enfermedades transmitidas por vectores como malaria (3), fiebre amarilla (4) y enfermedad de Chagas $(5,6)$. En 1998 se reportó en la ciudad de Leticia la presencia del mosquito Aedes albopictus (Skuse, 1895) (7), considerado eficiente vector de arbovirus al hombre. Con respecto a Aedes aegypti, a pesar de contar con

\footnotetext{
Correspondencia:

Yesika del Carmen Rojas, Secretaría de Salud Departamental, Seccional Amazonas, Carrera 11 № 3-96, Leticia, Amazonas, Colombia. Teléfono: (578) 5927295 y 592 7248, extensión 110

yesikarmen@yahoo.com.ar,ydrojasg@unal.edu.co embrochero@unal.edu.co

Recibido: 18/12/07; aceptado:08/09/08
}

registro para la especie en las provincias fronterizas con Perú (Caballococha en Ramón Castilla) (8), hasta el desarrollo de este estudio no se había registrado esta especie en el departamento (9). En Tabatinga, Brasil, ciudad fronteriza con Leticia, sólo se ha registrado la presencia de Ae. albopictus (10).

El Grupo de Enfermedades Transmitidas por Vectores de la Secretaría de Salud Departamental del Amazonas desarrolla estrategias para vigilar la introducción de Ae. aegypti en la región y la expansión de la población de Ae. albopictus a otras áreas del departamento. Esto es importante porque en este departamento la fiebre amarilla es endémica, Ae. aegypti se ha registrado como un eficiente transmisor urbano de esta enfermedad en Colombia (11) y no se han registrado casos de dengue en el departamento (12), por lo que las poblaciones humanas de sus comunidades son muy susceptibles a enfermar por dengue. Para ello, realiza levantamiento de índices de infestación por Aedes en localidades con conglomerados humanos y lleva a cabo programas educativos en diferentes sectores.

El casco urbano del corregimiento La Pedrera contaba con una larvitrampa ubicada en uno de los muelles del casco urbano para vigilar la presencia de especies de Haemagogus spp., vectores de fiebre amarilla, enfermedad que en el departamento es endémica. Esta larvitrampa no había registrado la presencia de $A e$. aegypti. Se determinó que las muestras entomológicas previas procedentes de esta larvitrampa y recibidas por la Unidad de Entomología de la Secretaría de Salud Departamental del Amazonas en marzo de 2006, correspondían a Culex nigripalpus. Sin embargo, se inició el levantamiento de índices de infestación larvaria teniendo en cuenta la sospecha de casos clínicos compatibles con dengue en esta localidad. Posteriormente, este diagnóstico fue descartado. 


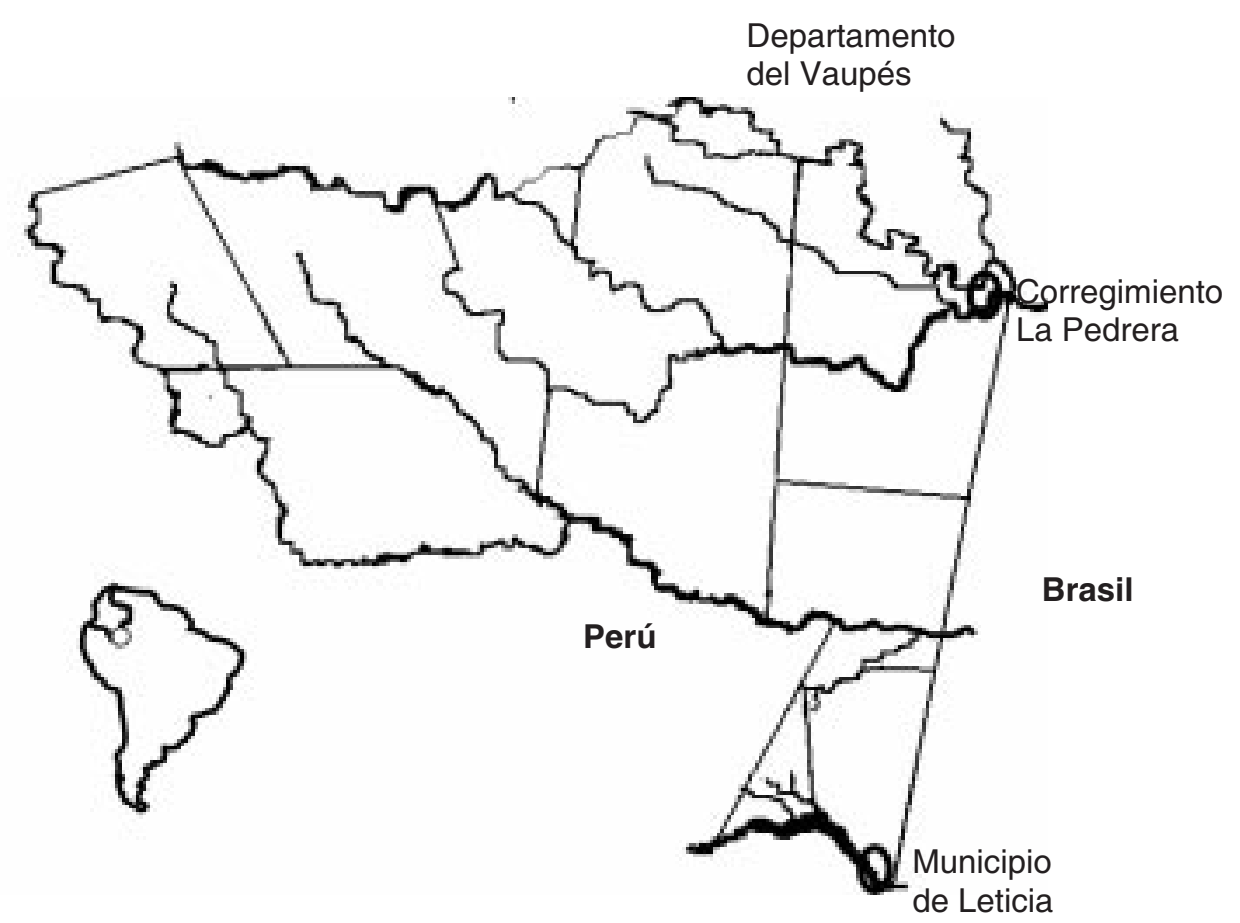

Figura 1. Departamento del Amazonas, Colombia. Esquema de la ubicación del municipio de Leticia, capital departamental, y del corregimiento de La Pedrera.

El presente estudio registra la presencia del mosquito Ae. aegypti en el casco urbano del corregimiento de La Pedrera en el departamento del Amazonas, Colombia, y aproxima aspectos de la percepción de sus habitantes con respecto a la presencia de este vector.

\section{Materiales y métodos}

\section{Área de estudio}

El casco urbano del corregimiento de La Pedrera se ubica en el nororiente de la amazonia colombiana, a una altitud de $100 \mathrm{~m}$ sobre el nivel del mar, y sobre el margen del río Caquetá; sus coordenadas son $01^{\circ} 19^{\prime}$ latitud norte y $69^{\circ} 30^{\prime}$ longitud oeste. Presenta serranías estrechas, alargadas y algo sinuosas con alturas que no sobrepasan los $400 \mathrm{msnm}$ (13). El período de lluvias se extiende desde marzo hasta septiembre con promedios mensuales que varían entre 208 y $379 \mathrm{~mm}$. Enero y febrero son los meses más cálidos, con temperaturas que oscilan entre $25,2^{\circ} \mathrm{C}$ y $27,6{ }^{\circ} \mathrm{C}$, mientras que el mes más frío es julio, con registros entre $23,5^{\circ} \mathrm{C}$ y $24,4^{\circ} \mathrm{C}(13,14)$.
De acuerdo con los registros de la gobernación del Amazonas 2007, el casco urbano es el centro socioeconómico del corregimiento y cuenta con 403 habitantes representados por colonos e indígenas de diferentes etnias. Registra 98 predios ubicados en 11 manzanas, todos sin suministro de agua potable y con un sistema deficiente de recolección y eliminación de residuos. La pista aérea y la comunicación fluvial facilitan la comunicación permanente con otras zonas del país, como Villavicencio, Huila y Caquetá, lo cual permite un permanente y activo intercambio comercial.

\section{Levantamiento de índices de infestación por Aedes}

Se realizaron dos inspecciones directas en búsqueda de formas inmaduras de Ae. aegypti en el $100 \%$ de los predios del corregimiento de La Pedrera. La primera en abril de 2006 y otra posterior al desarrollo de las actividades de control, en mayo de 2006. Los predios incluyeron viviendas, colegios, establecimientos comerciales, iglesia y batallón militar. 
En cada uno, se inspeccionaron depósitos para agua, con y sin ella, ubicados en áreas peridomiciliarias y espacios domiciliarios como habitaciones, cocinas, baños y salas. En todos los casos se registró el tipo de criadero según correspondiera a: tanques bajos (albercas y canecas ubicados sobre el suelo), tanques altos (tanques o canecas situados en superficies por encima del suelo), llantas, plantas y elementos diversos. Estos últimos correspondieron a depósitos de agua de carácter temporal tales como floreros, materas y desechos artificiales. Un predio se consideró positivo a la presencia de Ae. aegyptisi, por lo menos, se registró una forma inmadura de la especie en alguno de los depósitos inspeccionados.

Las muestras entomológicas se recolectaron en frascos con etanol al $70 \%$, indicando lugar, fecha, cantidad de ejemplares y nombre del recolector. Todos los especímenes se aclararon y montaron en forma permanente en bálsamo de Canadá (15). Algunas formas inmaduras, particularmente pupas, se conservaron vivas en agua del mismo criadero donde fueron encontradas, hasta la emergencia del mosquito adulto. La determinación taxonómica del material entomológico se realizó a partir de sus caracteres morfológicos, usando micrómetro ocular en microscopio y estereomicroscopio, y siguiendo las claves taxonómicas disponibles (1618). El Laboratorio de Entomología del Instituto Nacional de Salud confirmó la determinación taxonómica en todos los casos. Los ejemplares permanecen en la colección de referencia de la Unidad de Entomología del departamento del Amazonas.

\section{Actividades de control vectorial para Ae. aegypti}

En todos los predios, se aplicó $1 \mathrm{~g}$ de Abate® 1\% SG (ingrediente activo temefos) por cada 10 litros de agua, como tratamiento focal en los depósitos de agua que tenían larvas, así como en recipientes que contuvieran agua que no fuera posible remover y pudieran ser criaderos potenciales para el vector.

Los elementos diversos que contenían formas inmaduras del vector se manejaron directamente con la comunidad, eliminando su contenido sobre tierra seca. Además, se realizaron actividades para el mejoramiento del relleno sanitario de La Pedrera, colocándole un techo y haciendo un agujero adicional para colocar la basura, incluyendo tasas sanitarias y desechos artificiales.

La evaluación de impacto de esta estrategia se llevó a cabo un mes después de la aplicación, mediante el segundo levantamiento de infestación larvaria. Concomitantemente, se realizó un control químico perifocal mediante la aplicación de cuatro ciclos (uno cada semana) del insecticida malatión, en el $90 \%$ de los predios dado que algunos residentes no permitieron la aplicación del insecticida en su vivienda porque tenían bebés o peces ornamentales.

Los ciclos de fumigación se aplicaron simultáneamente con el larvicida con el objetivo de eliminar el insecto del corregimiento de La Pedrera a partir del impacto en las siguientes generaciones que pudieran emerger de criaderos ubicados por fuera de los predios y que no fueron detectados durante el levantamiento de los índices clásicos de infestación de formas inmaduras de la especie, y también, con el de eliminar cualquier riesgo de transmisión del virus del dengue (ante la sospecha de diagnóstico).

Después de 24 horas de aplicado cada ciclo de aspersión, se inspeccionaron las viviendas en búsqueda de mosquitos adultos. Para ello, un operario entrenado del Grupo de Control de Enfermedades Transmitidas por Vectores adscrito a la Secretaría de Salud Departamental del Amazonas, inspeccionó todas las áreas internas de los predios, siguiendo el sentido inverso a las manecillas del reloj y utilizando un aspirador bucal o una red de tul para su captura. Cada área de la vivienda se inspeccionó por 10 minutos.

Durante el desarrollo de ambos tratamientos, se llevaron a cabo actividades de información como charlas y entrega de folletos a la comunidad, para el reconocimiento de las formas inmaduras $y$ adultos de Ae. aegypti, su importancia como transmisor de enfermedades, sus hábitos domiciliarios y la necesidad de dar un uso adecuado a los recipientes que constituyen sus principales criaderos. Esta información verbal y escrita corresponde a la entregada por Grupo de 
Control de Enfermedades Transmitidas por Vectores en todas las localidades del departamento para la vigilancia entomológica de insectos de interés médico.

\section{Encuesta sobre el vector de dengue en el casco urbano de La Pedrera}

En mayo de 2006, durante el segundo levantamiento de infestación por Aedes, se realizó una encuesta sobre la percepción de los habitantes del casco urbano de La Pedrera en relación con las actividades y la información ofrecida por el Grupo de Control de Enfermedades Transmitidas por Vectores sobre los especímenes encontrados en el domicilio de sus predios en abril de 2006.

Se entrevistó a una persona adulta que viviera permanentemente en la unidad muestral inspeccionada, para un total de 78 personas. La encuesta se hizo con base en una previamente aplicada en el casco urbano de la ciudad capital, Leticia, con preguntas cerradas de selección múltiple a partir de un lenguaje regional. La indagación estuvo dirigida hacia dos componentes principales: 1) reconocimiento de las formas inmaduras y su relación con el mosquito adulto Ae. aegypti, así como el reconocimiento de estas formas del insecto dentro de la vivienda y, 2) principales factores de riesgo para el hallazgo de Ae. aegypti en los predios en relación con el manejo del agua de consumo humano y de basuras.

\section{Resultados}

En el cuadro 1 se observan los resultados del levantamiento de índices aédicos realizado en abril de 2006, comparados con los datos que se obtuvieron durante el segundo, efectuado en mayo de 2006, es decir, después de las intervenciones de control. Se observó una disminución en los índices de infestación de vivienda, depósito y Breteau, después de las intervenciones. Los depósitos más representativos con respecto a la presencia de formas inmaduras de Ae. aegypti en ambos muestreos fueron los tanques bajos, como albercas y canecas para la recolección de agua lluvia. Igualmente, los depósitos de agua con carácter temporal, tales como floreros, materas y recipientes artificiales, constituyeron criaderos aptos para la especie (cuadro 2). La larvitrampa ubicada en el muelle del casco urbano, así como las axilas, los troncos de árboles y las plantas inspeccionadas fueron negativas para la presencia del vector.

Después de la aplicación del larvicida se encontraron tres larvas de Ae. aegypti de cuarto estadio en una caneca plástica con capacidad de 60 galones, la cual contenía agua lluvia y se encontraba cubierta con tapa, y dos larvas de esta especie de segundo y cuarto estadio en una caneca metálica para agua lluvia con capacidad de 60 galones, sin tapa, que se encontraba bajo techo. Igualmente, se encontró una larva de $A e$. aegyptide tercer estadio en una tasa sanitaria en desuso que se encontraba al aire libre a seis metros de una vivienda, con capacidad de ocho litros, sin tapa y con presencia de materia orgánica. Las larvas encontradas correspondieron a viviendas donde no se permitió la aplicación de adulticida. Por otra parte, no se encontraron mosquitos adultos en el interior de las viviendas 24 horas después de cada ciclo de fumigación.

Cuadro 1. Índices de infestación larvaria para Aedes aegypti en el corregimiento La Pedrera, Amazonas, 2006.

\begin{tabular}{lcc}
\hline Parámetros e índices & Abril/2006 & Mayo/2006 \\
\hline Número de viviendas inspeccionadas & 98 & 98 \\
Número de viviendas con presencia de larvas de Ae. aegypti & 29 & 3 \\
Número de depósitos inspeccionados & 446 & 454 \\
Número de depósitos con presencia de Ae. aegypti & 40 & 3 \\
Promedio de depósitos con agua por vivienda & 5 & 5 \\
Índice de infestación de vivienda (\%) & 29,6 & 3,1 \\
Índice de infestación de depósito (\%) & 9,0 & 0,7 \\
Índice de infestación de Breteau & 40,8 & 3,1 \\
\hline
\end{tabular}


Cuadro 2. Hábitats inspeccionados y hallados con larvas de Aedes aegypti en el Corregimiento La Pedrera, Amazonas, 2006.

\begin{tabular}{|c|c|c|c|c|c|c|c|c|c|c|}
\hline \multirow[b]{2}{*}{$\begin{array}{l}\text { Tipos de } \\
\text { criaderos }\end{array}$} & \multicolumn{5}{|c|}{ Abril de 2006} & \multicolumn{5}{|c|}{ Mayo de 2006} \\
\hline & \multicolumn{2}{|c|}{$\begin{array}{c}\text { Depósitos } \\
\text { inspeccionados }\end{array}$} & \multicolumn{2}{|c|}{$\begin{array}{l}\text { Depósitos con } \\
\text { formas } \\
\text { inmaduras de } \\
\text { Ae. aegypti }\end{array}$} & $\begin{array}{l}\text { Peso porcentual } \\
\text { de depósitos } \\
\text { positivos con } \\
\text { respecto al total } \\
\text { de depósitos } \\
\text { positivos } \\
\text { inspeccionados }\end{array}$ & \multicolumn{2}{|c|}{$\begin{array}{c}\text { Depósitos } \\
\text { inspeccionados }\end{array}$} & \multicolumn{2}{|c|}{$\begin{array}{c}\text { Depósitos con } \\
\text { formas } \\
\text { inmaduras de } \\
\text { Ae. aegypti }\end{array}$} & $\begin{array}{l}\text { Peso porcentual } \\
\text { de depósitos } \\
\text { positivos con } \\
\text { respecto al total } \\
\text { de depósitos } \\
\text { positivos } \\
\text { inspeccionados }\end{array}$ \\
\hline Tanques elevados & 108 & 24,2 & 3 & 2,8 & 7,7 & 98 & 21,6 & 0 & 0 & 0 \\
\hline Tanques bajos & 264 & 59,2 & 32 & 12,1 & 82,1 & 261 & 57,5 & 2 & 0,8 & 66,7 \\
\hline Llantas & 1 & 0,2 & 1 & 100 & 2,6 & 1 & 0,2 & 0 & 0 & 0 \\
\hline Árboles y plantas & 5 & 1,1 & 0 & 0 & 0 & 24 & 5,3 & 0 & 0 & 0 \\
\hline Diversos* & 68 & 15,2 & 3 & 4,4 & 7,7 & 70 & 15,4 & 1 & 1,40 & 33,3 \\
\hline Total & 446 & 100 & & 39 & & 454 & 100 & & & \\
\hline
\end{tabular}

* Depósitos de agua con carácter temporal tales como floreros, materas, recipientes artificiales y naturales

De las 78 personas que fueron encuestadas, $67,9 \%$ correspondió al sexo femenino y $32,1 \%$ al masculino, con un rango de edad comprendido entre 17 y 70 años. La media, la mediana y la moda fueron de 36 años. El rango de tiempo vivido de forma permanente en el área urbana de La Pedrera estuvo entre un mes y 65 años. Los oficios mencionados correspondieron a hogar $(42 \%)$, comerciante $(16 \%)$ y estudiante $(7 \%)$, en tanto que el porcentaje restante se refiere a diferentes oficios. Con respecto al nivel de escolaridad, el $24 \%$ hizo algún curso de primaria y el $23 \%$ la concluyó, en tanto que el $26 \%$ hizo algún curso de básica secundaria y sólo el 16\% la concluyó. El 2\% manifestó ser analfabeta.

Las etnias a las cuales pertenecen los indígenas encuestados fueron: yucuna (12\%), miraña y tanimuca ( $6 \%$ cada una), macuna y matapi ( $4 \%$ cada una), andoque, carijona y huitoto ( $3 \%$ cada una), baraza, bora, cocama, inca, letuama, muinane, puinabe y ticuna ( $1 \%$ cada una). El $50 \%$ restante de las entrevistas correspondió a colonos no pertenecientes a etnia alguna.

La comunidad, en general, reconoce las formas inmaduras del mosquito en los recipientes de su domicilio. El $60,3 \%$ de la población ha visto organismos que se mueven en los depósitos con agua permanente situados en las viviendas, y de éstos, el $64 \%$ los denomina larvas, el $9 \%$ crías de zancudos y el $1 \%$ los denomina culebrilla o renacuajos. Sin embargo, el $4 \%$ no les concede denominación alguna. De los encuestados que relacionan al mosquito adulto con las formas inmaduras de los criaderos artificiales en los domicilios, $21 / 39(42 \%)$ de los colonos y 12/39 (31\%) de los miembros de la comunidad indígena manifestaron que la información había sido recibida del personal de salud, en tanto que el $17 \%$ indicó que había aprendido por acceso a la educación y el $22 \%$ que había observado por experiencia propia el fenómeno de la metamorfosis.

El $92 \%$ de la población percibe al mosquito indistintamente como cualquier zancudo y se encontró que el $6 \%$ cree que corresponde al género Anopheles sp., y el 1\%, a la especie Ae. albopictus. La comunidad considera que los zancudos encontrados en su vivienda transmiten enfermedades: malaria, $22 \%$; dengue, $22 \%$; fiebre amarilla, 4\%; cualquiera de éstas, $4 \%$, y afecciones como ronchas, diarreas y problemas en los huesos, $4 \%$. No obstante, el $45 \%$ no asocia a estas entidades como transmitidas por vectores de enfermedades; este concepto era generalizado entre los colonos (18/39) y entre los indígenas $(17 / 39)$. En este sentido, cinco personas manifestaron haber enfermado alguna vez de dengue, tres indígenas (una mujer y un hombre entre 10 y 44 años y una mujer mayor de 45 años) y dos colonos (una mujer y un hombre entre 10 y 44 años), quienes mencionaron que adquirieron la enfermedad en los departamentos de Caquetá, Casanare, Meta y Guaviare. Estas personas manifestaron que fueron diagnosticadas en un servicio de salud y que los síntomas más relevantes fueron fiebre y cefalea. 
Tanto los colonos (32/39) como los indígenas (32/ 39) manifestaron que suelen utilizar el servicio de recolección o quemar la basura, mientras que tan sólo los colonos (7/39) entierran los desechos. Sin embargo, durante la verificación en sus domicilios se encontraron 71 depósitos catalogados como inservibles, los cuales son desechados por la comunidad en áreas baldías del domicilio (patio trasero o solar). De éstos, 39\% correspondieron a botellas; $38 \%$, a recipientes como tarros de pintura y diferentes frascos; $13 \%$, a recipientes plásticos y galones viejos; $4 \%$, a artículos de cocina como bandejas, ollas, platos, vasos; $3 \%$, a bebederos de animales y $2 \%$, fueron baldes y sanitarios en desuso.

El total de colonos e indígenas almacenaban agua permanentemente mediante la tenencia de canecas con capacidad mayor de 200 litros, usadas para capturar el agua lluvia. No obstante, sólo el $61 \%$ de las pertenecientes a indígenas y el $72 \%$ propiedad de colonos, se encontraron tapadas totalmente durante la inspección. Todos los encuestados afirmaron lavar semanalmente, con agua y cepillo, tanto las canecas como las albercas. De estas últimas, pertenecientes a colonos, se encontraron 4/39 cubiertas totalmente con tapa y algunas de ellas (4/39) se encontraron abandonadas sin uso. Finalmente, sólo se encontró un florero durante la inspección de las 39 viviendas de colonos, en tanto que se encontró una llanta expuesta a la intemperie en las 39 viviendas de los indígenas.

\section{Discusión}

Hasta el año 2005 no se había registrado la presencia del mosquito Ae. aegypti en el departamento del Amazonas. Este hallazgo constituyó un resultado indirecto del sistema regular de vigilancia entomológica para determinar rangos de expansión de población y domiciliación de Ae. albopictus, especie introducida en Leticia y reportada desde 1998. Ae. aegypties dispersado por el hombre a través del transporte pasivo de huevos, larvas o adultos, ya sea por vía aérea, marítima o terrestre $(19,20)$. Es probable, entonces, que el hallazgo de la especie en el corregimiento de La Pedrera esté relacionado con la afluencia de comercio constante con zonas que registran altas infestaciones de la especie, particularmente, los departamentos de Meta y Huila.

El mosquito encontró condiciones climáticas y ecológicas aptas para su establecimiento, específicamente canecas y tanques utilizados para el almacenamiento de agua lluvia, así como recipientes descartados que son dejados a la intemperie por parte de la comunidad, aspectos culturales arraigados principalmente en los colonos. Aunque se sabe que los índices clásicos de infestación aédica, como los usados en este estudio, no permiten correlacionar el número de mosquitos adultos que produce cada criadero (2126), se observa una preferencia de Ae. aegypti por depositar sus huevos en recipientes artificiales, lo cual permitiría definir estrategias de reducción de las fuentes de cría de la especie a partir de programas diseñados teniendo en cuenta los aspectos culturales propios de las etnias y de los colonos $(27,28)$.

Se podría aprovechar la percepción de riesgo que la comunidad tiene con respecto a los zancudos en general, pero enfatizar sobre la relación entre las formas inmaduras y el mosquito adulto. En este contexto, es importante que a través de los programas comunitarios enfocados a cambios de conducta y estrategias de gestión integrada desarrollados a nivel regional para dengue, se incorpore la visión de salud ecosistémica donde "la salud no es únicamente la ausencia de enfermedad sino también, la medida en que un individuo o grupo sea capaz, por un lado, de realizar aspiraciones y satisfacer necesidades y, por el otro, de cambiar o enfrentar a su entorno" (29). Esta integración permitiría incorporar sistemas de vigilancia activa por parte de la comunidad mediante un proceso elaborado donde diversos actores e instituciones participen y converjan a partir de sus identidades culturales.

Debido al riesgo de ingreso del virus del dengue al casco urbano de La Pedrera por viajeros comerciantes y ante la alta susceptibilidad de la población, particularmente indígena, es importante que el personal de salud tenga claridad sobre los criterios de definición de caso probable de dengue. Teniendo en cuenta que este personal es itinerante (médicos rurales), la estrategia debe 
fortalecerse mediante el sistema educativo formal e informal, y este último, mediante la vinculación de acciones en los roles propios de la comunidad.

En este sentido, la participación de la mujer juega un papel importante en las estrategias de cambios conductuales tendientes a mejoras en salud pública por parte de una comunidad. El $68 \%$ de la población en el casco urbano de La Pedrera corresponde al sexo femenino $y$, de éste, un gran porcentaje se dedica a labores del hogar, lo que permitiría definir cambios conductuales para la reducción de riesgo de dengue a partir de actividades cotidianas de organización del hogar, particularmente, lo que tiene que ver con la proliferación de sitios de cría aptos para el vector, como el almacenamiento de agua lluvia y el manejo de recipientes de desecho utilizando el servicio de recolección de basuras. Esta aproximación es característicamente válida en La Pedrera porque la comunidad no ha sido aún objeto de prácticas de tipo paternalista, como es el suministro permanente de larvicida en sus tanques y canecas con agua.

Aunque luego de las intervenciones de tipo químico los criaderos del vector pasaron de un comportamiento multifocal a uno monofocal (evidenciado por los valores del índice de Breteau del primero y segundo levantamiento de infestación por Aedes), los valores de infestación aédica se redujeron hasta alcanzar un bajo riesgo epidemiológico y la comunidad percibe un riesgo de enfermedad ante la presencia del insecto, debe quedar claro que los conocimientos (información) adecuados no necesariamente derivan en la práctica de medidas de prevención y, por lo tanto, la sola difusión resulta insuficiente si no se combina con intervenciones que estén dirigidas a modificar las prácticas y comportamientos humanos, acompañadas con el mejoramiento de los servicios básicos $(30,31)$. Es importante, entonces, que los programas de movilización activa de la comunidad se inicien con la identificación de los problemas y necesidades locales por parte de los habitantes y se soporten con "grupos de trabajo comunitario" donde estén representados los intereses de funcionarios y de usuarios de los servicios de salud, involucrando realmente a la comunidad desde la planificación, la implementación y la evaluación de sus actividades $(32,33)$.

Se ha observado que los mosquitos adultos de Ae. aegypti pueden escapar a la acción del control químico realizado dentro de las viviendas, desplazándose hacia áreas abiertas (34). Las viviendas del casco urbano de La Pedrera presentan amplias áreas abiertas (patio trasero o solar), las cuales son usadas por la comunidad para desechar elementos inservibles. Es posible que la infestación larvaria hallada después de las actividades de control efectuadas, se haya originado por la oviposición, en depósitos inservibles desechados en los solares, de hembras que lograron escapar de la acción adulticida del control químico y que no pudieron ser detectadas durante el muestreo después del tratamiento. Es prioritario que se continúe la vigilancia entomológica regular y sostenida con el propósito de mantener los índices de infestación clásicos para Ae. aegypti en valores inferiores a los considerados de riesgo para la transmisión de dengue. Sería conveniente, además, incluir la realización de índices de infestación que permitan determinar la productividad de los criaderos aptos para la especie con el propósito de focalizar los esfuerzos de prevención y control.

El hallazgo de Ae. aegypti en La Pedrera no solamente representa un riesgo para dengue, sino que, teniendo en cuenta las condiciones selváticas de la zona donde la fiebre amarilla es endémica, se podría constituir en un riesgo para la urbanización de esta patología. Es fundamental, entonces, fortalecer las actividades de vigilancia entomológica en las comunidades cercanas a esta área, así como de los corregimientos de Mirití, La Chorrera, Puerto Santander y el municipio de Leticia, con los cuales se mantiene comunicación por vía fluvial y aérea. Además, se debe propender por continuar con el trabajo conjunto y multidisciplinario entre la Secretaría de Salud Departamental del Amazonas y sus homólogos de Perú y Brasil.

\section{Agradecimientos}

A la Secretaría de Salud de Amazonas, especialmente al Grupo de Control de Enfermedades 
Transmitidas por Vectores, por el apoyo logístico y de personal auxiliar que realizó gran parte de las actividades en campo.

\section{Conflictos de intereses}

Los autores declaramos que no incurrimos en conflicto de interés alguno que pueda afectar los resultados de esta investigación.

\section{Financiación}

Este proyecto fue financiado por la Secretaría de Salud de Amazonas.

\section{Referencias}

1. Instituto Geográfico Agustín Codazzi. Diccionario geográfico de Colombia. Bogotá, D.C.: IGAC; 1996.

2. Departamento Nacional de Estadística DANE. Boletín Censo General 2005. Perfil La Pedrera Amazonas. [Fecha de consulta: 30 de noviembre de 2007]. Disponible en: http://www.dane.gov.co/files/ censo2005/perfiles/amazonas/la_pedrera.pdf

3. Pérez L, Suárez M, Murcia L, de la Hoz F, Olano V, Brochero $\mathbf{H}$, et al. La malaria en el Amazonas: conocimientos, prácticas, prevalencia de parasitemia y evaluación entomológica en mayo de 1997. Biomédica. 1999;19:93-102.

4. Castillo O, Chaparro P, García I, Idárraga I, Izquierdo V, Otálvaro J, et al. Situación de las enfermedades transmisibles objeto de vigilancia intensificada en salud pública, Colombia, 2002. Inf Quinc Epidemiol Nac. 2002;7:463-74.

5. Gualdrón L, Brochero H, Arévalo C, Pérez L, Suárez M, Olano V. Hallazgo de algunos vectores de la enfermedad de Chagas en el departamento del Amazonas y sus implicaciones en salud pública. Revista Colombiana de Entomología. 2001;27:121-7.

6. Molina J, Gualdrón L, Brochero H, Olano V, Guhl F. Distribución actual e importancia epidemiológica de las especies de triatominos (Reduviidae: Triatominae) en Colombia. Biomédica. 2000;20:344-60.

7. Vélez I, Quiñones M, Suárez M, Olano V, Murcia LM, Correa E, et al. Presencia de Aedes albopictus en Leticia, Amazonas, Colombia. Biomédica. 1998;18:192-8.

8. Pan American Health Organization PAHO/WHO. Second Meeting to Establish a Surveillance Network for Emerging Infectious Diseases in the Amazon Region. Documento técnico. Tarapoto (Perú): PAHO/HCP/ HCT/143/99; 1999. p. 56.

9. Olano V, Padilla J, Sáenz R, Morales A, Pinzón E, Ferro C, et al. Distribución de Aedes aegypti en Colombia, 1997. Inf Quinc Epidemiol Nac. 1998;3:94-6.
10. Fé NF, Barbosa M, Alecrim W, Guerra M. Registro da ocorrência de Aedes albopictus em área urbana do município de Manaus, Amazonas. Revista de Saúde Pública. 2003;37:674-5.

11. Gast-Galvis A. Historia de la fiebre amarilla en Colombia. Bogotá: Instituto Nacional de Salud; 1978.

12. Instituto Nacional de Salud. Sistema Nacional de Vigilancia en Salud Pública, SIVIGILA 2005-2007. Bogotá, D.C.: Instituto Nacional de Salud; 2007.

13. Instituto Geográfico Agustín Codazzi. Zonificación ambiental para el plan modelo colombo-brasilero (Eje Apaporis-Tabatinga: PAT). Santafé de Bogotá, D.C.: Linotipia Bolívar; 1997. p. 45.

14. Universidad Nacional de Colombia. La Amazonia colombiana y sus recursos, proyecto radargramétrico del Amazonas. Bogotá: Universidad Nacional de Colombia; 1979. p. 590.

15. Martínez I. Técnicas básicas de anatomía microscópica y de morfometría para estudiar los insectos. Boletín SEA. 2002;30:187-95. [Fecha de consulta: 14 de abril de 2008]. Disponible en: http:// entomologia.rediris.es/aracnet/9/metodologias/ tecnicas/index.htm

16. Cova-García P, Sutil E, Rausseo JA. Mosquitos de Venezuela. Volumen II. Caracas: Publicaciones del Ministerio de Sanidad y Asistencia Social; 1966.

17. Forattini O. Culicidología médica. Volumen 2. Identificación, biología y epidemiología. São Paulo: Universidad de São Paulo; 2002. p. 860.

18. Rueda L. Pictorial keys for the identification of mosquitoes (Diptera: Culicidae) associated with dengue virus transmisión. Zootaxa. 2004;589:1-60.

19. Salvatella R. Aedes aegypti, Aedes albopictus (Diptera, Culicidae) y su papel como vectores en las Américas. La situación de Uruguay. Rev Med Uruguay. 1996; 12 : 28-36.

20. Marquetti, M, Suárez S, Bisset J, Leyva M. Reporte de hábitats utilizados por Aedes aegypti en Ciudad de La Habana, Cuba. Rev Cubana Med Trop. 2005;57:159-61.

21. Tun-Lin W, Kay B, Barnes A, Forsyth S. Critical examination of Aedes aegypti indices: correlations with abundance. Am J Trop Med Hyg. 1996;54:543-7.

22. Sánchez L, Vanlerberghe V, Alonso L, Marquetti M, Guzman M, Bisset J, et al. Aedes aegypti larval indices and risk for dengue epidemics. Emerg Infect Dis. 2006;12:800-6.

23. Focks D, Chadee D. Pupal survey: an epidemiologically significant surveillance method for Aedes aegypti: an example using data from Trinidad. Am J Trop Med Hyg. 1997;56:159-67.

24. Barrera R, Amador M, Clark G. Use of the pupal survey technique for measuring Aedes aegypti (Diptera: 
Culicidae) productivity in Puerto Rico. Am J Trop Med Hyg. 2006;74:290-302.

25. Morrison A, Gray K, Getis A, Astete H, Sihuincha M, Focks D, et al. Temporal and geographic patterns of Aedes aegypti (Diptera: Culicidae) in Iquitos, Perú. J Med Entomol. 2004;41:1123-42.

26. Guzmán M, Kouri G. Dengue: an update. Lancent Infect Dis. 2002;2:33-42.

27. Ooi E, Goh K, Gubler D. Dengue prevention and 35 years of vector control in Singapore. Emerg Infect Dis. 2006;12:887-93.

28. Kay B, Vu S. New strategy against Aedes aegypti in Vietnam. Lancet. 2005;365:613-7.

29. Ole Nielsen $\mathbf{N}$. Enfoques ecosistémicos para la salud humana. Reports in Public Health. 2001;17:69-75.

30. Navarrete-Espinosa J, Acevedo-Vales J, HuertaHernández E, Torres-Barranca J, Gavaldón-Rosas D. Prevalencia de anticuerpos contra dengue y leptospira en la población de Jáltipan, Veracruz. Salud Pública Mex. 2006;48:220-8.

31. Benítez-Leite S, Machi ML, Gibert E, Rivarola K. Conocimientos, actitudes y prácticas acerca del dengue en un barrio de Asunción. Rev Chil Pediatr. 2002;73:64-72.

32. Toledo-Romaní ME, Baly-Gil A, Ceballos-Ursula E, Boelaert M, van der Stuyft P. Participación comunitaria en la prevención del dengue: un abordaje desde la perspectiva de los diferentes actores sociales. Salud Pública Mex. 2006;48:39-44.

33. Toledo ME, Vanlerberghe V, Baly A, Ceballos E, Valdes L, Searret M, et al. Towards active community participation in dengue vector control: results from action research in Santiago de Cuba, Cuba. Trans $\mathrm{R}$ Soc Trop Med Hyg. 2007;101:56-63.

34. Castro M, Quintana N, Quiñones M. Evaluating two pyrethroids in dengue vector control in Putumayo, Colombia. Rev Salud Pública. 2007;9:106-16. 